\title{
AVALIAÇÃO E APLICAÇÃO DE TESTES PARA A DETECÇÃO DA AUTOCORRELAÇÃO ESPACIAL USANDO MARCADORES GENÉTICOS
}

\author{
Evaluation and application of tests for the detection of spatial autocorrelation \\ using data genetics marks
}

\author{
João Marcos Louzada ${ }^{1}$, Eduardo Bearzoti², Dulcinéia de Carvalho ${ }^{3}$
}

\begin{abstract}
RESUMO
O índice $I$ de Moran é a ferramenta usual para se medir a intensidade da autocorrelação espacial em dados de marcadores genéticos. A estatística $I$ é assintoticamente normalmente distribuída, podendo ser avaliada como desvios da normal padrão mediante o suposto-N (aproximação normal). Porém, para pequenos números de populações $(\mathrm{m}<8)$, deve-se aplicar o teste de aleatorização de Mantel (suposto-R), desenvolvido por Mantel (1967). Assim, por meio do presente estudo, buscou-se avaliar o desempenho de ambos os testes no que tange às taxas de erro tipo I e o poder dos mesmos. Estes foram avaliados via simulação de Monte Carlo, em que analisaram-se, sob a $\mathrm{H}_{0}$, as situações $\{\mathrm{p}=0,1, \mathrm{p}=0,25 \mathrm{e} \mathrm{p}=0,5\}$ de freqüências alélicas médias, para as quais consideraram-se os números $\{\mathrm{m}=5,10,25$ e 50$\}$ de populações, sendo que variou-se, para cada população, o número de indivíduos em $\{\mathrm{n}=1,2,5$ e 10$\}$. Em relação à hipótese alternativa (com padrão espacial), além das mesmas situações simuladas em $\mathrm{H}_{0}$, avaliou-se o comportamento destes critérios de testes segundo a variação da amplitude na freqüência alélica média local em $\{A=0,1 ; 0,2 ; 0,5 ; 0,8$ e 1,0\}. Desta forma, pôde-se analisar a performance dos testes em questão quanto ao grau de variabilidade das freqüências alélicas médias geradas sobre uma superfície linear, em função do espaço geográfico, por meio de diferentes inclinações da mesma. O teste da aproximação normal foi considerado melhor com $\{\mathrm{m} \leq 10\}$ populações, quando combinado com as ponderações inverso da distância e inverso da distância ao quadrado, em ambos os níveis de significância, 1\% e 5\%. Porém, o mesmo não deve ser aplicado associado com a ponderação vizinho mais próximo. Com $\{\mathrm{m} \geq 25\}$, deve-se aplicar o teste de Mantel em qualquer das situações simuladas.
\end{abstract}

Termos para indexação: Autocorrelação espacial, simulação Monte Carlo, $I$ de Moran, freqüências alélicas, teste de Mantel.

\begin{abstract}
Moran's $I$ index is the usual tool to measure the intensity of the spatial autocorrelation in genetic markers data. $I$ statistics is asymptotically normally distributed and it may be evaluated as standard normal deviations (assumption-N, normality). However, for small numbers of populations ( $\mathrm{m}<8)$, the Mantel's randomness test (assumption-R) developed by Mantel (1967) should be applied. Thus, this study was done to evaluate the performance of both tests accordding to type I error rate sand their power. They were evaluated via Monte Carlo simulation, in which, the situations of average allelic frequencies, $\{\mathrm{p}=0,1, \mathrm{p}=0,25$ and $\mathrm{p}=0,5\}$ were analyzed under $\mathrm{H}_{0}$. Number for populations varying from $\{\mathrm{m}=5,10,25$ and 50$\}$ were taken into account and for each population, the number of individuals in $\{\mathrm{n}=1,2,5,10$ and 30$\}$ was varied as well. As regards to the alternative hypothesis (with spatial pattern), in addition to these same situations simulated in $\mathrm{H}_{0}$, the behavior of these criteria of tests was evaluated according to the variation of the amplitude in the average local allelic frequency in $\{A=0,1 ; 0,2 ; 0,5 ; 0,8$ e 1,0$\}$. Therefore, the performance of the test studied could be analyzed as the degree of variability of the average frequencies generated on a linear surface, related to the geographic space and by means of it's different slopes. The normal approximation was considered better withpopulations as combined with the weighing systems inverse of the distance and inverse of the distance squared in both levels of significance $1 \%$ and $5 \%$. The same should not be applied associated with the nearest neighbor weighing. With , Mantel's test should be applied in any of the situations simulated.
\end{abstract}

Index terms: Spatial autocorrelation, Monte Carlo simulation, Moran's I, allelic frequencies, Mantel's test.

(Recebido para publicação em 12 de março de 2004 e aprovado em 7 de dezembro de 2005)

\section{INTRODUÇÃO}

A principal justificativa do uso dos métodos da estatística espacial está na problemática da independência. Dados independentes são uma suposição conveniente, a qual torna mais simples a inferência. Porém, modelos que envolvem dependência estatística são freqüentemente mais realistas (Cressie, 1993). Quando um indivíduo apresenta alguma qualidade que, provavelmente, se faz presente em indivíduos vizinhos, diz-se que o fenômeno exibe autocorrelação espacial.

A análise de autocorrelação espacial tem sido aplicada como ferramenta para se fazer inferências de processos que estão subjacentes nos padrões espaciais observados de variação em freqüências alélicas. Sokal e Jacquez (1991), por meio de estudo de simulação aplicando a análise de autocorrelação espacial, recomendam o uso

\footnotetext{
'Mestre em Estatística e Experimentação Agropecuária Departamento de Ciências Exatas/UFLA - Escola Agrotécnica Federal de Colatina - BR 259 km 70 - Cx. P. 256 - 29709910 - Colatina, ES - jmarlo@bol.com.br

2 Professor da Universidade Federal de Lavras/UFLA - Cx. P. 3037 - 37200-000 - Lavras, MG.

${ }^{3}$ Professora da Universidade Federal de Lavras/UFLA - Cx. P. 3037 - 37200-000 - Lavras, MG.
} 
da análise de autocorrelação para detectar processos microevolucionários em populações naturais.

Quando se analisam padrões de superfície para explicar a variabilidade de frequiências alélicas por meio de estatística espacial, deve-se, então, representar esta variabilidade em um espaço tridimensional, cujos eixos X e $\mathrm{Y}$ denotam as componentes espaciais, sobre as quais associa-se o eixo $\mathrm{Z}$ das frequiências alélicas (Figura 1). Dessa forma, define-se o tipo básico de dados para a análise espacial de diferenciação de populações locais, em que a variável (frequiência alélica) mensurada em diversos pontos amostrais, cuja posição geográfica é conhecida (DinizFilho, 1998). Na Figura $1^{\mathrm{a}}$, sugere-se uma superfície contínua, em forma de um gradiente de variação, que pode ser interpolada em relação aos valores de Z, como mostra a Figura 1b. Esta superfície ajustada ilustra razoavelmente bem o comportamento real da variação das frequiências alélicas, de modo que este fenômeno dependa apenas da posição geográfica $\mathrm{S}_{\mathrm{i}}$ em qualquer parte da região $\mathrm{D}$, tal que $\left\{\mathrm{s}_{\mathrm{i}} \in \mathrm{D}\right\}$.

Se essa superfície for bem ajustada, refletirá a existência de um padrão espacial de variação. Evidentemente nem sempre esta superfície é necessariamente uma estrutura real, pois as populações locais podem não se distribuir de forma contínua sobre o espaço; porém, deve refletir a dinâmica espacial de um processo microevolutivo que atua sobre as freqüências alélicas. De fato, a existência de um padrão espacial implica no fato de que é possível encontrar uma explicação biológica única para a variação (ou pelo menos grande parte dessa) nas diversas populações locais.
Em genética de populações, estudos de padrões espaciais em estruturas genéticas são usualmente analisados por meio do índice I de Moran (Cliff e Ord, 1973). Define-se esta estatística, segundo Moran (1950), como sendo:

$$
I=\frac{\mathrm{m} \sum_{\mathrm{i}=1}^{\mathrm{m}} \sum_{\mathrm{j}=1}^{\mathrm{m}} \tau_{\mathrm{i}} \tau_{\mathrm{j}} \mathrm{w}_{\mathrm{ij}}}{\mathrm{S}_{0} \sum_{\mathrm{i}=1}^{\mathrm{m}} \tau_{\mathrm{i}}^{2}}
$$

em que $m$ é o número de populações amostradas, $\tau_{\mathrm{i}}=\left(\mathrm{z}_{\mathrm{i}}-\overline{\mathrm{z}}\right)$ e $\tau_{\mathrm{j}}=\left(\mathrm{z}_{\mathrm{j}}-\overline{\mathrm{z}}\right)$ são as variáveis frequiências alélicas das populações i e j centradas na média; $\mathrm{w}_{\mathrm{ij}}$ é o elemento da matriz quadrada e simétrica $\mathbf{W}$, com dimensão mxm, a qual expressa a relação espacial entre as $m$ populações e $\mathrm{S}_{0}$ é o somatório dos elementos da matriz simétrica de pesos espaciais $\mathbf{W}$.

A interpretação do índice $I$ é análoga ao coeficiente de correlação de Pearson, sendo então suas estatísticas, na maior parte dos casos, restritas ao intervalo $[-1,+1]$. Finalmente, valores em torno de zero indicam que não há autocorrelação espacial.

A composição da matriz de continuidade, $\mathbf{W}$, é bastante flexível, constando de esquema s mais simples como a medida de conectividade (vizinhança), bem como por meio de esquema de pesos generalizados, tais como o recíproco da distância, o recíproco da distância ao quadrado, ou alguma outra potência: $\left\{1 / \mathrm{d}, 1 / \mathrm{d}^{2}, \ldots, 1 / \mathrm{d}^{\mathrm{r}}\right\}$. Especificamente, em genética de populações, em que as amostras se distribuem de forma irregular, é muito comum utilizar a rede de Gabriel para definir os pesos binários (Gabriel e Sokal, 1969).

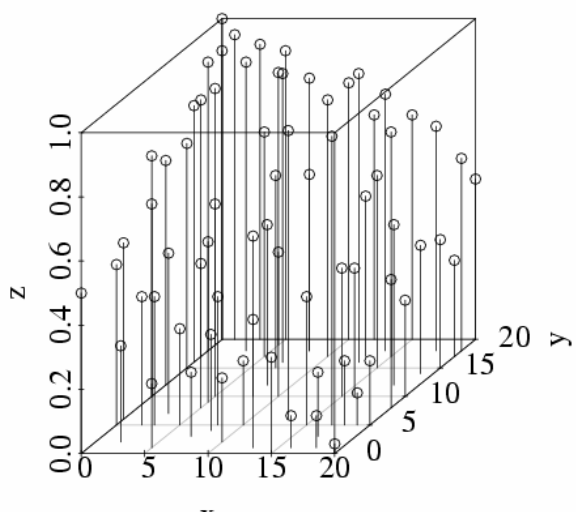

(a) Variação de frequiência alélica

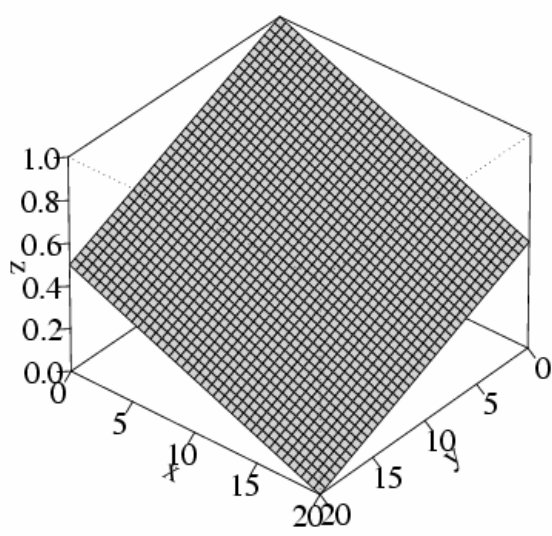

(b) Superfície ajustada

FIGURA 1 - Superfície de freqüência simulada sobre espaço geográfico com variação clinal. 
A significância dos momentos de $I$ podem ser avaliados sob $\mathrm{H}_{0}$, por duas suposições básicas: 1) suposto- $N$ (aproximação normal): assume-se que os dados são realizações de $m$ amostras independentes e identicamente distribuídas, proveniente de uma ou mais populações normais; 2 ) suposto- $R$ (teste de aleatorização de Mantel): admite-se que as observações são realizações aleatórias de $m$ amostras independentes, tomadas de uma ou mais populações, cuja função de distribuição é desconhecida. Para maiores detalhes sobre a fundamentação teórica relativa ao $I$ de Moran, consultar Cliff e Ord $(1973,1981)$.

Entretanto, dada a importância de se detectar padrões espaciais em estudos de genética de populações, teve-se por objetivo com esse estudo avaliar o desempenho (poder) dos critérios de teste de aleatorização de Mantel (suposto-R) e da aproximação normal (suposto-N) para detectar a presença de autocorrelação espacial, considerando que os mesmos são usualmente utilizados para se testar a significância do índice $I$ de Moran.

\section{MATERIAL E MÉTODOS}

Avaliou-se a performance dos critérios de teste de Mantel (teste de aleatorização) e da aproximação normal por meio de simulação de Monte Carlo, em aplicações simuladas de estudos de genética de populações.

Ambos os testes foram avaliados em diferentes condições de padrão espacial, considerando diferentes números de populações $\{m=5,10,25$ e 50$\}$, variando os tamanhos (n) de amostras, com $\{1,2,5,10,30\}$ indivíduos por população; estabeleceram-se ainda, os valores nominais de significância $\alpha=1 \%$ e $\alpha=5 \%$ para os testes. Para simular os dados, concebeu-se uma superfície linear sobre um plano cartesiano, de maneira a representar uma variação clinal ao longo de populações hipotéticas dispostas no espaço geográfico, sendo este, por sua vez, representado pelo plano cartesiano. Sem perda de generalidade, a superfície foi construída sobre este plano tendo como valor mínimo no ponto do domínio $(0,0)$ e o valor máximo em $(10,10)$. Dessa forma, o valor médio da superfície era encontrado nos pontos pertencentes à reta ligando as coordenadas $(0,10)$ e $(10,0)$.

Assim, definida uma freqüência alélica média $\mu$ ao longo das populações, e uma amplitude de variação $A$ (diferença entre o máximo e o mínimo), tem-se a equação da superfície dada por:

$$
E\left(p_{i}\right)=\mu-\frac{A}{2}+\frac{A}{20} x_{i}+\frac{A}{20} y_{i}
$$

com $0 \leq \mathrm{p}_{\mathrm{i}} \leq 1$ e sendo $\mathrm{x}_{\mathrm{i}}$ e $\mathrm{y}_{\mathrm{i}}$ as coordenadas da população i no plano cartesiano. Conforme sugere a notação $\mathrm{E}\left(\mathrm{p}_{\mathrm{i}}\right)$, a superfície buscou justamente representar a freqüência alélica média de uma população na posição $\left(\mathrm{x}_{\mathrm{i}}, \mathrm{y}_{\mathrm{i}}\right)$ do espaço.

Para gerar populações sob a hipótese de nulidade (ausência de padrão espacial), bastava atribuir o valor $A=0$ em (2). Dessa forma, obtém-se uma superfície paralela ao plano cartesiano, com uma dada freqüência alélica média $\mu$. Sob $\mathrm{H}_{0}$, considerou-se $=0,5 ; 0,25$ e 0,1 .

Em presença de dependência espacial, as seguintes condições foram avaliadas:

$$
\{\mu=0,5 ; \mathrm{A}=1,0 ; 0,8 ; 0,5 ; 0,2 \text { e } 0,1\},\{\mu=0,25 ; \mathrm{A}=0,5 ; 0,2 \text { e } 0,1\},\{\mu=0,1 ; \mathrm{A}=0,2 \text { e } 0,1\}
$$

Considerando-se todas as combinações possíveis entre os parâmetros, foram abordadas 211 situações.

Objetivando-se verificar a melhor ponderação para compor a matriz de distâncias, $\mathbf{W}$, combinou-se, com cada situação citada as ponderações inverso da distância (ID), inverso da distância ao quadrado (IDQ) e a vizinho mais próximo (VMP).

Com cada uma dessas ponderações, em cada uma das 5.000 amostras geradas, o teste de Mantel e o teste da aproximação normal do $I$ de Moran foram feitos, para julgar a hipótese $\mathrm{H}_{0}$ de ausência de padrão espacial. Para o teste de Mantel, um total de 2.000 permutações foram feitas. $\mathrm{O}$ teste baseado na aproximação normal foi realizado utilizando-se o suposto R. Assim, em cada amostra e para cada matriz de ponderação, a seguinte estatística foi calculada: $\mathrm{Z}_{I}=\frac{I-\mathrm{E}(I)}{\sqrt{\operatorname{var}(I)}}$, na na qual $\mathrm{E}(\mathrm{I})$ é a esperança do $I$ de Moran, definida como, $\mathrm{E}(I)=-\frac{1}{\mathrm{~m}-1}$ em que $m$ é o tamanho da amostra, conforme definido em (1).

Tanto no critério de Mantel quanto na aproximação normal, testes unilaterais à direita foram realizados para julgar a hipótese de ausência de padrão espacial.

Ao longo das 5.000 amostras, calculou-se a

Ciênc. agrotec., Lavras, v. 30, n. 2, p. 206-213, mar./abr., 2006 
proporção de amostras com as quais foi rejeitada. Intervalos de confiança exatos também foram calculados para tais proporções (adaptado de Leemis e Trived (1996)). Neste estudo, utilizou-se intervalos com de confiança.

Além das taxas de rejeição de $\mathrm{H}_{0}$, o valor médio e a variância do $I$ de Moram, ao longo das 5.000 amostras, também foram calculados para cada um dos três critérios de ponderação.

\section{RESULTADOS E DISCUSSÕES}

Para as condições analisadas via simulação de Monte Carlo, em relação às ponderações inverso da distância e inverso da distância ao quadrado, verificou-se que a variância do índice I de Moran, foi diretamente afetada pela amplitude de variação média $(A)$, tendendo a aumentála. Por outro lado, a magnitude de $\mathrm{m}$, contrariamente, também influenciou esta mesma variância, tendendo a diminuí-la.

No que se refere à ponderação ID (Figura 2), as taxas de rejeição de $\mathrm{H}_{0}$, segundo o critério de Mantel, tenderam a se apresentar estatisticamente diferentes dos níveis nominais de significância; notadamente com $\{m=5\}$, as taxas foram estatisticamente menores que 0,05 , quando este era o nível nominal e, quando o nível nominal era 0,01 , as taxas foram um pouco superiores. Nos casos de $\{m=10\}$, quando significativas, as taxas estiveram abaixo dos níveis nominais (Figura 3a e 3d). Este fato denota um certo conservadorismo do critério de teste. Deve-se notar, contudo, que a partir de $\{m=25\}$, as taxas de rejeição de foram quase sempre estatisticamente iguais aos valores nominais, indicando um controle adequado do erro tipo I (Figura 3a). Já os testes da aproximação normal, com $\{\mathrm{m}=5\}$, foram mais conservadores, tanto com os níveis iguais a 0,05 como 0,01. Ou seja, neste último nível, o teste promoveu um melhor controle do erro tipo I que o critério de Mantel, que apresentou taxas superiores a 0,01 com $\{\mathrm{m}=5\}$ (Figura 2a). Por meio da Figura 3a, nota-se que com tamanhos amostrais superiores a 5 populações, as taxas dos testes baseadas na normalidade apresentaram uma tendência de serem superiores aos níveis de significância, embora não muito distantes destes. Especialmente nos casos com $\{\mathrm{m}=10\}$ e , o teste da aproximação normal foi claramente superior ao critério de Mantel.

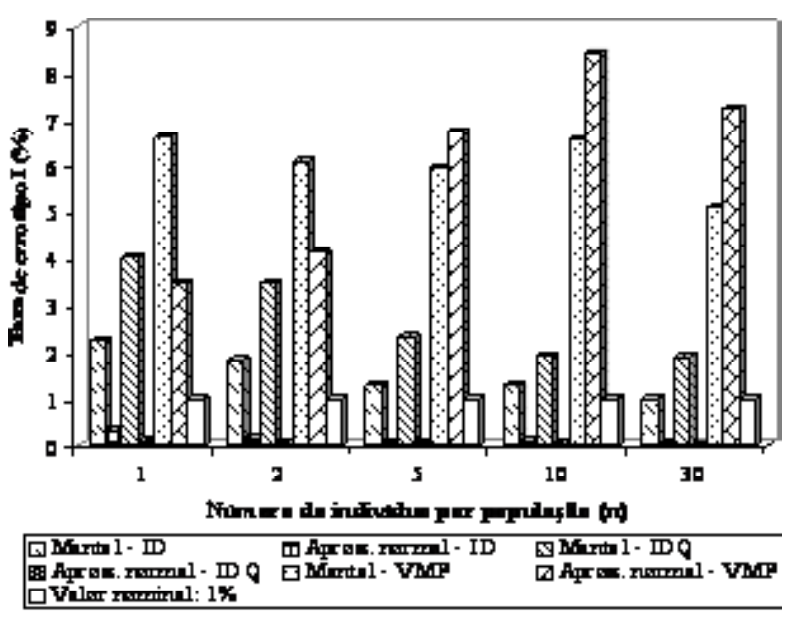

(a) taxa de erro tipo $I, \operatorname{com} \alpha=1 \%$ e $p=0,25$

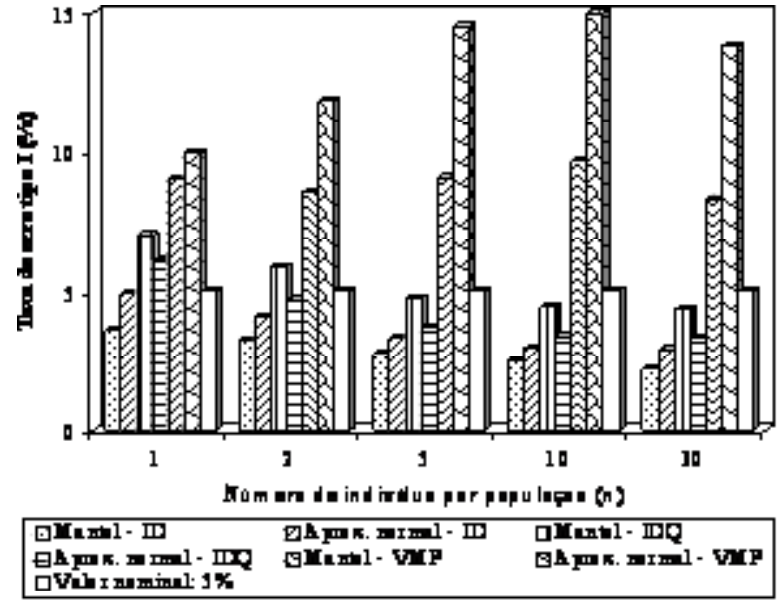

(b) taxa de erro tipo I para $\alpha=5 \%$ e $p=0,5$

FIGURA 2 - Taxa de erro tipo I do teste de Mantel e da aproximação normal em função do número de indivíduos por população $(n)$, para $m=5$. 
Estes resultados sugerem que, com amostras pequenas (iguais ou menores que 10), utilizando-se o inverso da distância como ponderação e com nível de significância 0,05 a aproximação normal é superior ao teste de Mantel, a julgar exclusivamente pelo controle de erro tipo I. Com $\{m=25\}$ ou mais, o critério de Mantel já apresenta um controle satisfatório deste tipo de erro, devendo ser preferido. A aproximação normal só apresentou, consistentemente, taxas estatisticamente iguais aos valores nominais com $\{\mathrm{m}=50\} \mathrm{e}$, mesmo assim, com $=0,05$.

Quanto à ponderação inverso da distância ao quadrado, com $\{\mathrm{m}=5\}$, o teste de Mantel teve uma leve tendência de as taxa serem estatisticamente superiores ao valor nominal 0,05 ; ao contrário do que ocorreu com a ponderação ID, conforme mostra a Figura $2 \mathrm{~b}$. Com $m$ igual ou superior a 10, houve maior semelhança de tendências, tendo o teste Mantel também apresentado um controle satisfatório do erro tipo I, a partir de $\{\mathrm{m}=25\}$ populações (Figura 3b). Em relação ao teste baseado na aproximação normal, o comportamento foi semelhante à ponderação ID, tendo apenas observado, um controle estatisticamente diferente dos níveis de significância.

No que tange à ponderação vizinho mais próximo, $\mathrm{o}$ teste de Mantel teve bastante semelhança com as tendências observadas na ponderação IDQ, ou seja, com $\{\mathrm{m}=5\}$ as taxas de rejeição de $\mathrm{H}_{0}$ foram superiores aos valores nominais de nível de significância. Com $\{m=10\}$, tais taxas foram, quando significativas, em geral, inferiores, e, a partir de $\{\mathrm{m}=25\}$, podese dizer que o teste de Mantel promoveu um controle satisfatório do erro tipo I (Figura 3c).

$\mathrm{O}$ mesmo não pode ser dito no tocante à aproximação normal, apresentando taxas muito elevadas de rejeição de $\mathrm{H}_{0}$, na grande maioria das situações. $\mathrm{Ou}$ seja, a julgar pelos resultados encontrados, se o pesquisador optar pelo vizinho mais próximo como fator de ponderação, então não deve utilizar a aproximação normal como critério de teste, em função de seu desempenho muito insatisfatório de controle do erro tipo I (Figuras 2 e $3 c$ ).
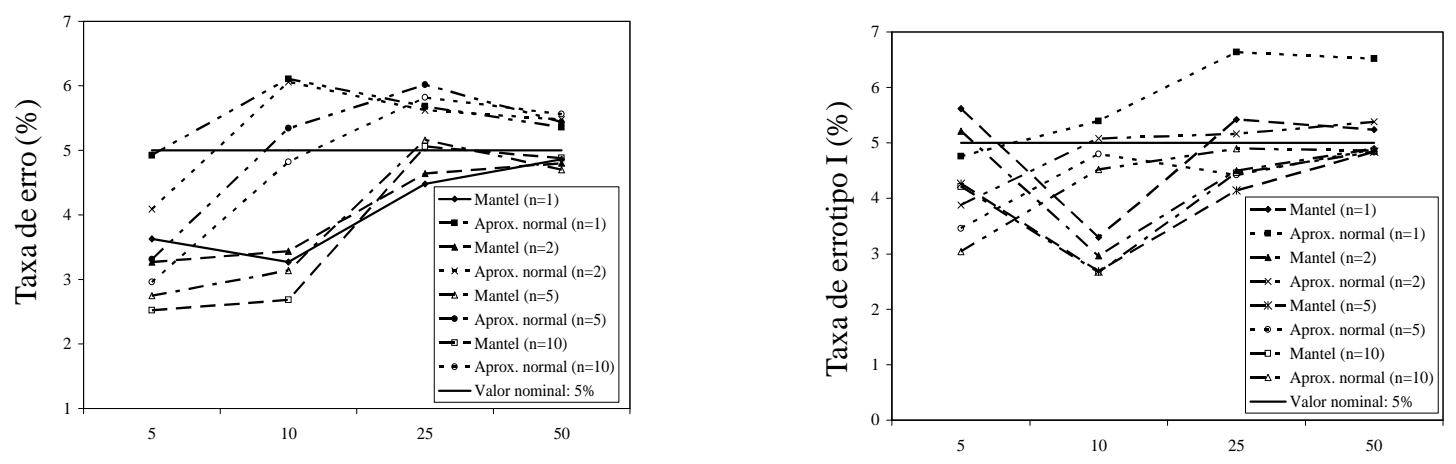

(a) taxa de erro para $\alpha=5 \%, p=0,25$ e ID

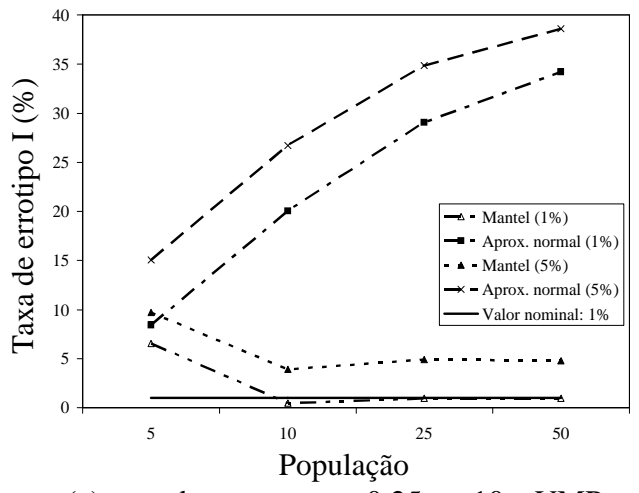

(c) taxa de erro para $\mathrm{p}=0,25, \mathrm{n}=10$ e VMP

(b) taxa de erro para $\alpha=5 \%, p=0,5$ e IDQ

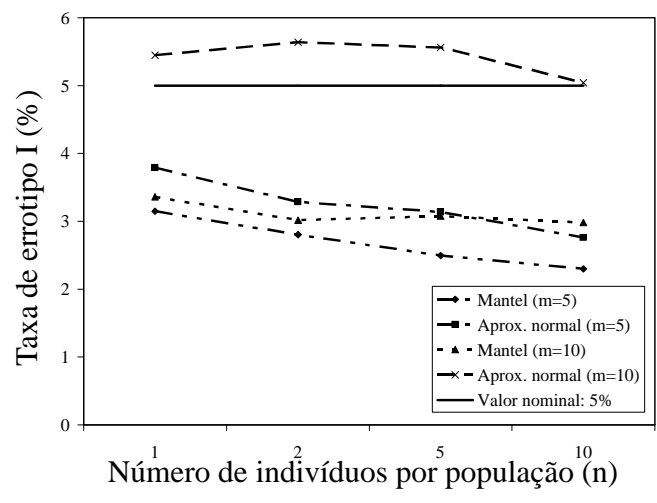

(d) taxa de erro para $\alpha=5 \%, p=0,5$ e ID

FIGURA 3 - Taxa de erro tipo I dos testes de Mantel e da aproximação normal em função da população (m) e do número de indivíduo por população (n).

Ciênc. agrotec., Lavras, v. 30, n. 2, p. 206-213, mar./abr., 2006 
Sob a hipótese de dependência espacial, no tocante à ponderação ID, o teste de Mantel, apresentou uma clara tendência de as taxas de rejeição de $\mathrm{H}_{0}$ aumentarem com o aumento da amplitude $A$. Ou seja, quanto maior a inclinação do plano de variação clinal, maior o poder do teste. Esta tendência foi observada em ambos os níveis de significância adotados (Figura 4a).

Chamam a atenção, os valores de poder muito baixos, com amostras pequenas $\{\mathrm{m}<10\}$.Tomandose o teste de Mantel com $\alpha=0,05$, observa-se que, na maioria dos casos, o poder esteve abaixo de 0,1 ; tendo atingido o valor $\mathbf{0 , 1 4 5 8}$ na situação de maior inclinação do plano de variação clinal. Mesmo com um tamanho de amostra dado por $\{\mathrm{m}=25\}$, o que pode ser considerado uma condição relativamente típica, o poder foi muito baixo com valores de $A$ intermediários. Na situação mais extrema $(A=1)$, o poder chegou a $\mathbf{0 , 3 9 9 9}$, com $\alpha=0,05$. Estes resultados sugerem que, em situações típicas, mesmo que haja considerável dependência espacial entre os indivíduos, o pesquisador terá dificuldades de detectar tal dependência, utilizando o índice $I$ de Moran associado ao teste de Mantel. Este aspecto é ainda agravado se for utilizado o nível de significância 0,01 , conforme mostram a Figuras $4 \mathrm{a}$ e $4 \mathrm{~b}$. Valores desejáveis de poder, como aqueles acima de 0,9 , só puderam ser observados com tamanhos totais de amostras grandes, como a partir das combinações $\{m=25, n=10\}$ e $\{m=50, n=5\}$. Tais tamanhos, no entanto, são pouco freqüentes na prática em genética, ver Figura 4c.

Conforme discutido anteriormente, o teste baseado na aproximação normal apresentou, em geral, um melhor controle da taxa de erro tipo I sob pequenas amostras $(\mathrm{m} \leq 10)$, portanto, é de é de especial interesse compará-lo como o teste de Mantel em tais condições. De fato, pode-se observar, na Figura 4d, que o poder da aproximação normal foi quase sempre maior que o teste de Mantel, com $\{m=5\}$ e $\{n=10\}$. Exceções foram observadas apenas com $\{m=5\}$ e $=0,01$. Assim, sugerese que, com amostras pequenas, a aproximação normal deva ser preferida ao critério de Mantel. No entanto, é importante salientar que, mesmo com a aproximação normal, os valores de poder foram marcadamente baixos, sob amostras pequenas.
É interessante notar que, com a ponderação IDQ, houve uma sensível redução de poder nos casos com $\{\mathrm{m}=50\}$ (Figura $4 \mathrm{~d}$ ). Este aspecto provavelmente se deve à pior especificação do fator de ponderação. Assim, mesmo com as amostras grandes, é importante que haja uma especificação adequada da ponderação a ser utilizada. Esta não é uma questão simples, sugerindo que estudos devam ser conduzidos no sentido de se elucidar como se dá o padrão de variação clinal. Na prática, é pouco provável que ele se dê por meio de uma superfície estritamente linear e, assim, a escolha da ponderação mais adequada não é óbvia.

A frequiência alélica média desempenhou grande influência sobre o poder. Isso se deve ao fato de que, quanto mais a frequiência alélica média se aproximar de zero, menor será a amplitude de variação possível entre o maior e o menor valor do plano clinal. Este é um aspecto que dificulta ainda mais a detecção de dependência espacial, pois nem sempre os locos marcadores apresentam alelos com frequiência próxima a 0,5 , conforme mostra a Figura 4e.

Em relação ao fator de ponderação vizinho mais próximo, os valores de poder também cresceram em função de $A$, mas também houve exceções. Comparando-se as taxas de poder do teste de Mantel com o produto igual a 50, nota-se uma redução do poder com $\{\mathrm{m}=50\}$, da mesma forma como observado com a ponderação inverso da distância ao quadrado. Este é um aspecto estatístico indesejável, pois sugere inconsistência, ou seja, aumento de taxas de erro tipo II, com o aumento do tamanho da amostra, se a ponderação especificada não é mais apropriada, conforme mostra a Figura 4f.

De uma maneira geral, pode-se dizer que, no teste de Mantel, a ponderação VMP promoveu valores de poder intermediários entre as ponderações ID e IDQ. Este fato sugere que o vizinho mais próximo seja uma ponderação um pouco mais robusta. No tocante ao teste da aproximação normal, os valores de poder sempre foram consideravelmente superiores com a ponderação VMP, em ambos valores nominais de significância (Figuras $4 \mathrm{~b}$ e $4 \mathrm{~d}$ ). Isso sugere superioridade deste critério, à primeira vista. Porém, uma vez que o controle do erro tipo I com a aproximação normal deixou a desejar, realmente não se deve recomendá-la, em absoluto, se o vizinho mais próximo for a ponderação escolhida. 


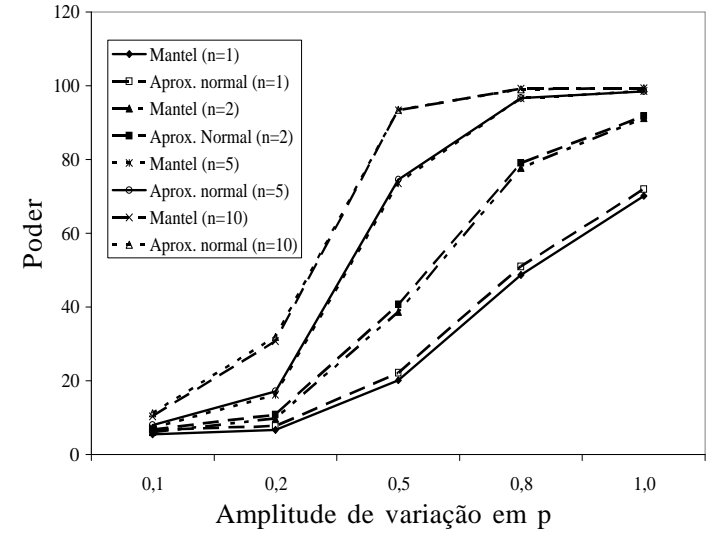

(a) poder em função de $A$ com ponderação ID, p=0,5, $\mathrm{m}=50$ e $\alpha=5 \%$

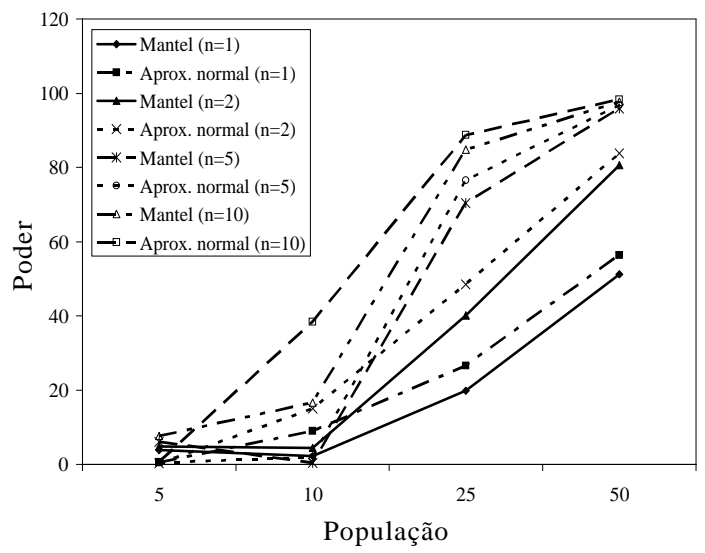

(c) poder em função da população $m$, com a ponderação ID, para $\mathrm{p}=0,5, \mathrm{~A}=1$ e $\alpha=1 \%$.

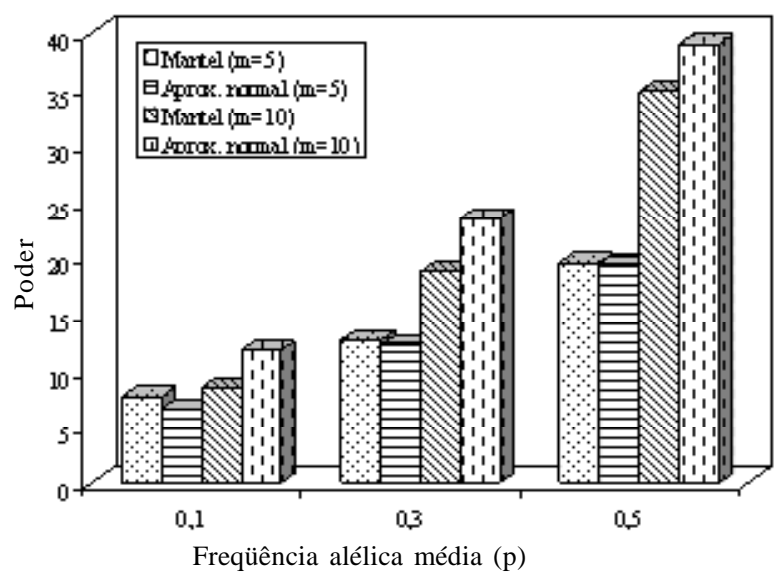

(e) poder em função de $\mathrm{p}, \mathrm{n}=10, \alpha=5 \%$, ponderação IDQ, em que considerou-se a maior amplitude de variação média (A).

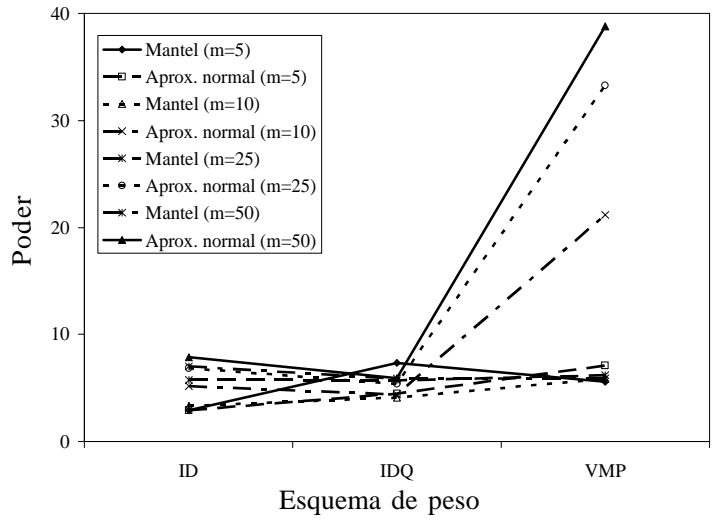

(b) poder em função das ponderações com $\mathrm{p}=0,5, \mathrm{n}=10$, $\mathrm{A}=1$ e $\alpha=5 \%$

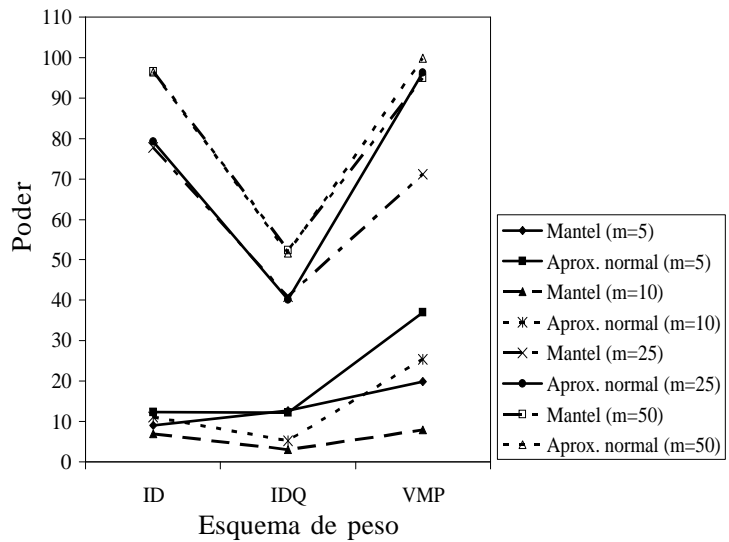

(d) poder em função das ponderações, para $\mathrm{p}=0,25, \mathrm{n}=10$, $\mathrm{A}=0,5$ e $\alpha=5 \%$.

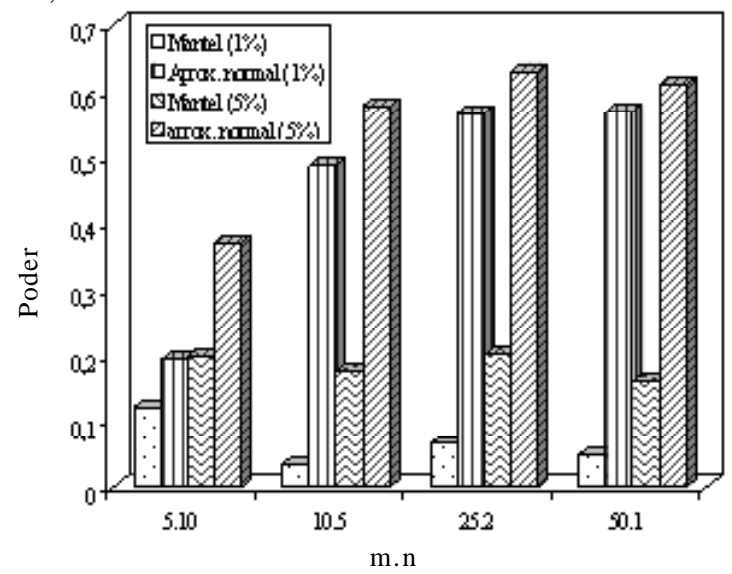

(f) poder em função de $\mathrm{m} . \mathrm{n}=50$, sendo $\mathrm{p}=0,25, \mathrm{~A}=0,5$, ponderação VMP e $\alpha=1 \%$ e $\alpha=5 \%$.

FIGURA 4 - percentuais de poder dos testes de Mantel e da aproximação.

Ciênc. agrotec., Lavras, v. 30, n. 2, p. 206-213, mar./abr., 2006 


\section{CONCLUSÕES}

Embora com baixo poder, o teste da aproximação normal deve ser preferido para as situações de pequenas amostras $(\mathrm{m} \leq 10)$, quando se utilizam os esquemas de ponderações inverso da distância e o inverso da distância ao quadrado, pois o mesmo controlou melhor as taxas de erro tipo I, para ambos os níveis de significância adotados.

Para o tamanho populacional maior ou igual a 25, o critério de Mantel teve melhor desempenho, pois controlou satisfatoriamente as taxas de erros. Somente para o caso $\{m=50\}$ o teste da aproximação normal obteve taxas de erros consistentes e estatisticamente iguais ao valores nominais, com o valor nominal $\alpha=0,05$.

Os poderes de ambos os testes foram fortemente influenciados pelas freqüências alélicas médias (ou frequiências alélicas intermediária), com as quais pode-se obter inclinações mais pronunciadas. Caso contrário, terse-á mais dificuldades em se detectar o padrão espacial.

Em relação ao fator ponderação inverso da distância ao quadrado, pôde-se notar uma queda no poder dos testes nos casos com $\{\mathrm{m}=50\}$. Isso é um forte indício de que mesmo para grandes amostras, deve-se ter muita cautela na escolha adequada do esquema de ponderação. Este fato sugere a importância de estudos que expliquem melhor como se dá o padrão de variação clinal. Na prática, é muito provável que este padrão espacial não se dê por meio de uma superfície rigorosamente linear. Assim, a escolha da ponderação adequada não é tão clara.

Em linhas gerais, o teste da aproximação normal não é recomendado em nenhuma das situações simuladas quando se utiliza o esquema vizinho mais próximo.

\section{REFERÊNCIAS BIBLIOGRÁFICAS}

CRESSIE, N. A. C. Statistics for spatial data. rev. edth. New York: Iowa State Univesity, 1993. 900 p. (A Wiley Interscience Publication).

CLIFF, A. D.; ORD, J. K. Spatial autocorrelation. London: Pion Press, 1973. 178 p.

DINIZ-FILHO, J. A. F. Análise de autocorrelação espacial. Inferências microevolutivas e aplicações em genética de populações. Séries Monográficas, n. 6, p. 89-138, 1998.
ENDLER, J. A. Geographic variation, speciation and clines. Princeton: Princeton University Press, 1977.

GABRIEL, K. R.; SOKAL, R. A new statistical approach to geographic variation analysis. Systematic Zoology, Washington, v. 18, n. 3, p. 259-278, 1969.

ISSAKS, E. H.; SRIVASTAVA, R. M. Applied geostatistics. New York: Oxford University Press, 1989. 561p.

KOENIG, W. D. Spatial autocorrelation of ecological phenomena. Trends in Ecology \& Evolution, London, v. 14, n. 1, p. 22-26, Jan. 1999.

LEGENDRE, P. Spatial autocorrelation. Ecology, Washington, v. 74, n. 6, p. 1659-1673, Sept. 1993.

LEGENDRE, P.; FORTIN, M. J. Spatial pattern and ecological analysis. Vegetatio, Dordrecht, v. 80, n. 2, p. 107138, June 1989.

LEEMIS, L. M.; TRIVED, K. S. A comparison of approximate interval estimators for the bernoulli parameter. The American Statistician, Alexandria, v.50, n.1, p. 63-68, Feb. 1996.

MANLY, B. F. J. Randomization and Monte Carlo Methods in biology. London: Chapman and Hall, 1991. 399 p.

MANTEL, N. The detection of disease clustering and a generalized regression approach. Cancer Research, Birmingham, v. 27, n. 2, p. 209-202, 1967.

MORAN, P. A. F. Notes on continous stochastic phenomena. Biometrika, London, v. 37, n. 2, p.17-23, 1950.

SOKAL, R. R. Testing statistical significance of geographic variation patterns. Systematic Zoology, Washington, v. 28, n. 2, p. 227-232, 1979.

SOKAL, R. R.; JACQUEZ, G. M. Testing inferences about microevolutionary processes by means of spatial autocrrelation analysis. Evolution, Washington, v. 45, n. 1, p. 152-168, Feb. 1991. 